\title{
Alina Cherry, Claude Simon: Fashioning the Past by Writing the Present
}

Andera Goulet

\section{OpenEdition}

1 Journals

\section{Édition électronique}

URL : http://journals.openedition.org/ccs/2536

DOI : $10.4000 /$ ccs. 2536

ISSN : 2558-782X

Éditeur :

Presses universitaires de Rennes, Association des lecteurs de Claude Simon

\section{Édition imprimée}

Date de publication : 30 septembre 2019

Pagination : 273-274

ISBN : 978-2-7535-7795-4

ISSN : 1774-9425

Référence électronique

Andera Goulet, "Alina Cherry, Claude Simon: Fashioning the Past by Writing the Present ", Cahiers Claude Simon [En ligne], 14 | 2019, mis en ligne le 30 septembre 2020, consulté le 10 octobre 2020. URL:

http://journals.openedition.org/ccs/2536 ; DOI : https://doi.org/10.4000/ccs.2536 


\section{Alina Cherry, Claude Simon : Fashioning the Past by Writing the Present, Madison, NJ, Fairleigh Dickinson University Press, 2016, 203 p.}

L'affirmation bien connue de Claude Simon que «l'on n'écrit [... jamais que ce qui se passe au présent de l'écriture » fournit à Alina Cherry la base d'une réflexion soutenue et approfondie sur les rapports esthétiques, philosophiques, et éventuellement éthiques entre le passé et le présent dans l'œuvre du romancier. Le mot clé "simultanéité " se dégage de son étude comme un horizon à la fois incontournable et inaccessible, le rêve de capter l'immédiat de la perception sans tomber dans l'illusion mimétique de l'écriture. En disant refuser la fonction référentielle du langage, Simon soulève un paradoxe apparent : comment concilier une théorie de la "présentification " avec une ouvre qui tourne de manière obsessionnelle autour des thèmes " passéistes " tels que la généalogie familiale, la mémoire individuelle ou collective, les événements historiques, guerriers, et épiques? Cherry explore cette question en se servant des théories de la temporalité de Ricœur et des travaux importants menés par des spécialistes de Simon - Britton, CalleGruber, Carroll, Dällenbach, Duffy, Janssens, Sarkonak, Viart - qui ont amplement retracé les tensions temporelles au cœur de la pratique littéraire de Simon. Les synthèses méticuleuses et nuancées que fait Cherry de ces discussions critiques sont déjà d'une grande utilité pour les chercheurs et pour les lecteurs anglophones qui découvrent Simon. (Notons non seulement que l'auteure traduit les textes critiques et littéraires en anglais pour cette édition, mais aussi qu'elle propose une bibliographie très complète, avec une section dédiée aux entretiens et conférences de Simon). Mais la contribution la plus importante de ce livre se trouve dans l'emploi original que fait Cherry des travaux de Deleuze et Guattari, notamment leur concept du devenir-animal, afin de comprendre le langage simonien en des termes qui dépassent les oppositions binaires réductrices de passé/présent, réalité/écriture, formalisme/engagement.

Cherry relie d'abord la polysémie du texte simonien - ses jeux de mots, ses " plumes " et ses « feuilles ", ses " racines » et ses " elles/ailes » - aux ramifications multiples du rhizome, que Deleuze et Guattari ont décrit dans leur Mille plateaux. En prenant comme point de départ le motif de l'arbre (botanique et généalogique) dans L'Acacia, Cherry démontre de manière convaincante que la structure en arborescence du roman s'oppose à toute hiérarchie discursive stabilisante. L'écriture de Simon exemplifie ainsi la fonction contestataire du rhizome deleuzien, non seulement dans la thématique de la plupart de ses romans, mais aussi dans leur structure : dans Histoire, par exemple, Cherry souligne que la mise en page typographique, les « brise-phrase " disjonctives, et la circularité auto-réflexive contribuent à cet effet " rhizomatique " qui démolit les concepts d'unité, de centre et de linéarité. En ceci, l'approche de Cherry entre en résonance avec 
celle de Christy Wampole, qui sonde la "rhizosphère » dans son excellent livre paru récemment : Rootedness : the Ramifications of a Metaphor (Chicago, 2016). Mais là où Wampole aborde les écrits de Ponge, Claudel, Valéry, Weil, et Sartre, l'expertise de la simonienne férue qu'est Cherry lui permet de faire une plongée profonde dans l'œuvre de l'écrivain pour y examiner les conséquences de l'arborescence textuelle en la reliant à tout un discours critique sur le positionnement de Simon vis-à-vis des nouveaux romanciers. Sa prédilection stylistique, par exemple, pour les rectifications, les répétitions, les rajustements ["non, ce n’est pas cela..."] a moins à voir avec le présentisme descriptif d'un Robbe-Grillet qu'avec le "bégaiement " que Deleuze et Guattari associent au " devenir-mineur " d'une écriture non-hiérarchique.

C'est dans ce contexte que Cherry érige sa belle lecture de la thématique de la chasse chez Simon comme expression de l'éthique du "devenir-animal » de Deleuze et Guattari. Cherry suggère qu'à travers plusieurs romans, le motif de l'animal traqué représente non pas une régression vers un état primitif et non-langagier mais une " involution " productrice, une création textuelle qui sert à contester le discours anthropocentrique de la maîtrise. Quand le narrateur-soldat de la cavalerie dans L'Acacia se trouve assailli dans une embuscade militaire, il "devient " chien; à quatre pattes, ses réflexes canins servent de réponse aux événements cataclysmiques de l'Histoire et son instinct animal devient stratégie de survie. Dans Les Géorgiques, le " devenir-rat » de O. en Catalogne et les métamorphoses figurales du frère de L.S.M. en loup, lapin, ou renard, fonctionnent aussi comme "lignes de fuite " pour des êtres empêtrés dans le bourbier de la guerre. En outre, l'indifférenciation du corps du cheval mort dans La Route des Flandres entraîne une décomposition à la fois corporelle et textuelle, à travers un processus de déterritorialisation comme étape nécessaire au " devenir » d'un langage non-majoritaire. Cherry n'est pas la première à relever des éléments deleuziens dans l'œuvre de Simon (elle cite Patrick Longuet et Guy Scarpetta ${ }^{1}$ ), mais elle développe davantage son analyse et la relie aux débats sur l'engagement politique du romancier. Tout en dénonçant une écriture idéologique à la Sartre ou Malraux, Simon crée un langage subversif dans sa propre vitalité « rhizomatique».

Cherry développe cette thèse " deleuzo-guattarienne " surtout dans son quatrième et dernier chapitre, mais elle mérite d'être mise au premier plan. D'une certaine manière, on pourrait lire les autres chapitres - avec leurs études minutieuses des thèmes simoniens plus reconnus, tels la trace, l'archive, l'histoire, et la mémoire - comme des étapes amenant habilement cette thèse fondamentale. Grâce à cette approche, qui révèle des lectures approfondies et sensibles aux nuances du langage simonien, et met en évidence des synthèses intelligentes d'une série de débats sur le passé et le présent dans l'œuvre du romancier, le livre de Cherry apporte une contribution significative aux études simoniennes.

Andrea Goulet

1. Voir également I. Yocaris et D. Zemmour, "Vers une écriture rhizomatique : style et syntaxe dans La Bataille de Pharsale de Claude Simon ", Semiotica, n 181, août 2010, p. 283-312. 